\title{
Preparation of stable polymeric grafted layers on poly(ethylene terephthalate) by thermal annealing
}

\author{
Peerasak Paoprasert $^{\mathrm{a}, *}$, Warisara Boonthong ${ }^{\mathrm{a}}$, Chanatip Kookarinrat ${ }^{\mathrm{a}}$, Nuanphun Chantarasiri $^{\mathrm{b}}$ \\ a Department of Chemistry, Faculty of Science and Technology, Thammasat University, \\ Pathumthani 12121 Thailand \\ b Supramolecular Chemistry Research Unit, Department of Chemistry, Faculty of Science, \\ Chulalongkorn University, Bangkok 10330 Thailand
}

*Corresponding author, e-mail: peerasak@tu.ac.th

Received 29 Jan 2014

Accepted 11 May 2014

\begin{abstract}
This work presents a simple and versatile grafting method to prepare stable polymer layers on poly(ethylene terephthalate) (PET). Poly(styrene-co-2-hydroxyethyl methacrylate) was synthesized by free radical polymerization. The copolymer was grafted onto poly(ethylene terephthalate) via the hydroxyl groups upon thermal annealing. The percentage of copolymer grafting was studied as a function of copolymer composition, PET surface treatment, annealing time, and annealing temperature. The grafted polymer layer on PET remained stable when immersed in an organic solvent whereas the non-grafted polymer layers were completely desorbed within two weeks. In addition, poly(4-vinylpyridine-co-2hydroxyethyl methacrylate) was grafted onto PET to test the versatility of this grafting method. Control experiments were carried out to demonstrate that the grafting occurs through the available hydroxyl groups on the polymers and the surfaces. Based on these results, this grafting method offers a simple and versatile strategy to prepare stable polymeric layers on polymeric surfaces, and therefore, expands the tool box for functionalizing polymer surfaces.
\end{abstract}

KEYWORDS: coatings, adhesion, polymer grafting, hydroxyl linker

\section{INTRODUCTION}

The modification of polymeric substrates by grafting is an important route to modify a polymer surface properties and to introduce new functions, for example, biocompatibility ${ }^{1}$, adhesion ${ }^{2}$, friction ${ }^{3}$, lubrication $^{4}$, hydrophobicity ${ }^{5}$, or hydrophilicity ${ }^{6}$, to the substrates. There are two main approaches to graft polymers onto solid surfaces: grafting-from and graftingto ${ }^{7,8}$. In the grafting-from method, the monomers react with a surface activated by high energy irradiation or a chemical initiator forming polymer chains tethered to the surface. In the grafting-to approach, the pre-synthesized polymer chains are anchored onto the solid surface through linker groups. The graftingfrom method often yields high-density polymer layers, but has poor control over polymer molecular weight and distribution. In addition, it uses a large amount of starting materials, including the monomer and initiator (in the case of chemical grafting). Since the samples must be totally immersed in a solution, the graftingfrom method produces abundant chemical waste and a relatively small amounts of polymers grafted to the surface. In contrast, in the grafting-to method, the polymer is pre-synthesized so that the desired molecular weight and distribution can be obtained prior to grafting. Less chemical waste is generated as a thin layer of polymer can be directly transferred to the substrate before the grafting step. The linker groups however must be installed in the polymer chains and therefore the proper linkers must be chosen and the polymerization conditions must be compatible.

There are several linker groups for grafting organic materials onto inorganic substrates. For example, silanes ${ }^{9}$, carboxylic acids ${ }^{10}$, phosphonic acids ${ }^{11}$, and catechols ${ }^{12}$ are linkers for oxide surfaces whereas thiols are linkers for gold ${ }^{13}$. However, unlike the inorganic substrates, the polymeric substrates are more difficult to graft because most commodity polymers are inert or lack active groups such as surface hydroxyls or metal atoms, to react with those linkers. There are only a few reports on grafting polymers on polymeric substrates, for example, fluorinated silane grafted poly(vinylidene fluoride $)^{14}$, hydrophilic polymer-coated polydimethylsiloxane through silane linker ${ }^{15}$, and hepamine-coated polyurethane through catechol linker ${ }^{16}$. Grafting such polymers is difficult as the synthesis of these polymers requires several steps and the grafted polymeric layers may be unstable. There is a need therefore to develop new grafting methods for the preparation of stable polymeric layers on polymer surfaces. 
(a)


(b)
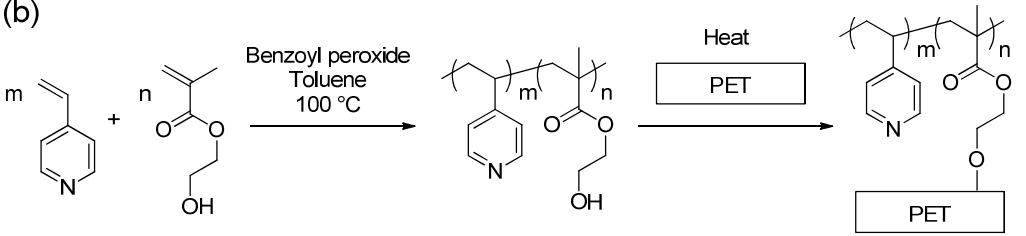

P(4VP-CO-HEMA)

P(4VP-co-HEMA)-grafted PET

Fig. 1 Synthesis and grafting on PET surface of (a) P(S-co-HEMA) and (b) P(4VP-co-HEMA).

Recently, hydroxyl groups have been used as surface linker to graft polymers ${ }^{17}$ and rheniumbipyridine dyes ${ }^{18}$ onto oxide substrates by thermal annealing. By simple heating at $140-160^{\circ} \mathrm{C}$, the hydroxyl groups of an organic material and oxide surface condense to form an ether linkage resulting in organic layers chemically bonded onto the surface. The organic layers show good stability against desorption in aqueous and organic solutions.

In this study, poly(ethylene terephthalate) (PET) was chosen as the polymeric substrate because it is a cheap, non-toxic, and chemically stable semicrystalline thermoplastic polymer ${ }^{19}$ widely used to fabricate several kinds of commercial articles. In addition, PET has been used for a variety of advanced technological applications ranging from electronic ${ }^{20}$ to biological devices ${ }^{21}$. Polymers based on styrene and 4-vinylpyridine were used to graft onto PET surfaces. The copolymers were characterized by nuclear magnetic resonance spectroscopy (NMR), Fourier transform infrared spectroscopy (FTIR), and gel permeation chromatography (GPC). The surface topography and composition of the grafted samples were characterized using atomic force microscopy (AFM) and Xray photoelectron spectroscopy (XPS), respectively. The stability of the grafted polymer layers in organic solvent was investigated.

\section{MATERIALS AND METHODS}

\section{Materials}

All chemicals were purchased from Sigma-Aldrich. Styrene, 2-hydroxyethyl methacrylate, and 4-vinylpyridine were purified by passing through silica gel columns. Benzoyl peroxide was recrystallized in methanol and dried at room temperature.

\section{Synthesis of copolymers}

The copolymers used in this work were synthesized using a conventional free radical polymerization method as follows (Fig. 1). To prepare poly(styreneco-2-hydroxyethyl methacrylate) (P(S-co-HEMA)), styrene ( $8 \mathrm{~g}), 2$-hydroxyethyl methacrylate $(1 \mathrm{~g}$ for $10 \mathrm{~mol} \%$ and $4 \mathrm{~g}$ for $40 \mathrm{~mol} \%)$, toluene $(10 \mathrm{ml})$, and benzoyl peroxide (30 mg) were added to a roundbottom flask. The reaction mixture was heated at $100{ }^{\circ} \mathrm{C}$ for $16 \mathrm{~h}$. Then the solution was cooled down, diluted with toluene, and precipitated in methanol twice. To prepare poly(4-vinylpyridine-co-2-hydroxyethyl methacrylate) (P(4VP-co-HEMA)), 4-vinylpyridine (8 g), 2-hydroxyethyl methacrylate (4 g), toluene $(10 \mathrm{ml})$, and benzoyl peroxide $(30 \mathrm{mg})$ were added to a round-bottom flask. The reaction mixture was heated at $100^{\circ} \mathrm{C}$ for $16 \mathrm{~h}$. Then the solution was cooled down, diluted with toluene, and precipitated in methanol twice.

\section{Grafting procedure}

PET samples were obtained as a thin sheet and cut into $0.5 \times 0.5 \mathrm{~cm}^{2}$ pieces. The average thickness of PET samples measured by a micrometre was $0.240 \pm 0.001 \mathrm{~mm}$. Prior to grafting, the PET samples were cleaned by ultrasonication in acetone, hexane, and deionized water (10 min each), and then dried at $70^{\circ} \mathrm{C}$ overnight in an oven. The PET samples were dipped in an ethyl acetate solution of copolymer $(0.2 \mathrm{mg} / \mathrm{ml})$ and air-dried. Then they were thermally annealed at temperatures from $100^{\circ} \mathrm{C}$ to $160^{\circ} \mathrm{C}$ for times from $6 \mathrm{~h}$ to $96 \mathrm{~h}$. After that the samples were ultrasonicated in dichloromethane $(3 \times 10 \mathrm{ml}$ 
(a)

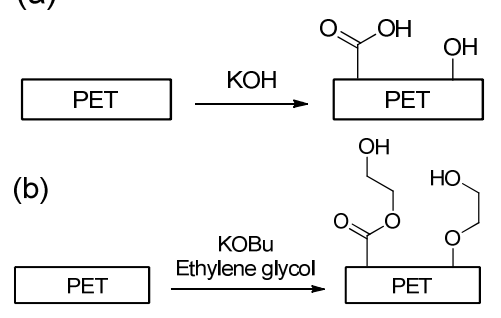

Fig. 2 Preparation of (a) $\mathrm{KOH}$-treated PET and (b) $\mathrm{KOBu}$ treated PET.

for 10 min each) and dried overnight at $70{ }^{\circ} \mathrm{C}$ until the weight was constant. A confirmation test was carried out by subjecting the sample to soxhlet extraction in chloroform overnight. It was found that the weight of the sample did not change. This test confirms that the ultrasonication in dichloromethane ( $3 \times 10 \mathrm{ml}$ for $10 \mathrm{~min}$ each) is sufficient to clean the samples. The percentage of grafting was determined as $\left(w_{2}-w_{1}\right) / w_{1}$, where $w_{2}$ is the weight of sample after grafting and $w_{1}$ is the weight of PET sample before grafting. The average percentage of grafting was calculated from at least five samples in each condition.

\section{Surface treatment procedures}

Two surface treatment methods, $\mathrm{KOH}$ and potassium tert-butoxide $(\mathrm{KOBu})$, were employed on PET samples following standard procedures from the literature $^{22}$ as follows (Fig. 2). Cleaned PET samples were heated in $1 \mathrm{M} \mathrm{KOH}$ solution at $80^{\circ} \mathrm{C}$ for $90 \mathrm{~min}$. The samples were then ultrasonicated in deionized water three times $(10 \mathrm{~min}$ each) and rinsed with acetone. The samples were then dried overnight at $70{ }^{\circ} \mathrm{C}$. To treat with $\mathrm{KOBu}$, cleaned PET samples were immersed into $25 \mathrm{ml}$ of $0.60 \mathrm{M} \mathrm{KOBu}$ solution in ethylene glycol. After $4 \mathrm{~h}$ of reaction at room temperature, the film was rinsed sequentially with ethylene glycol (two aliquots), water, $0.1 \mathrm{M} \mathrm{HCl}$, water (two aliquots), methanol, and hexane and then dried overnight at $80^{\circ} \mathrm{C}$.

\section{Stability test}

Three different PET samples were prepared for the stability test in organic solvent as follows. To prepare $\mathrm{P}(\mathrm{S}$-co-HEMA)-grafted PET, the cleaned PET samples were dipped in an ethyl acetate solution of $\mathrm{P}(\mathrm{S}$-co-HEMA) $(0.2 \mathrm{mg} / \mathrm{ml})$ and for thermally annealed at $130^{\circ} \mathrm{C}$ for $48 \mathrm{~h}$ (Fig. 3a). The samples were then ultrasonicated in dichloromethane $(3 \times 10 \mathrm{ml}$ for $10 \mathrm{~min}$ each) and dried overnight at $70^{\circ} \mathrm{C}$. To (a)



(b)

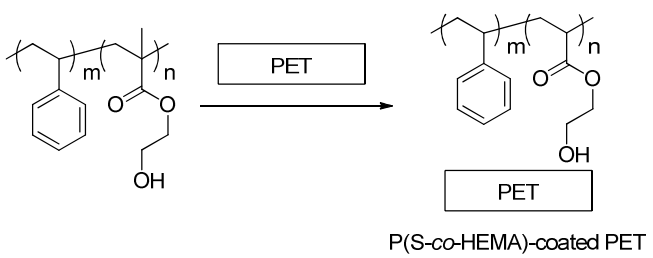

(c)

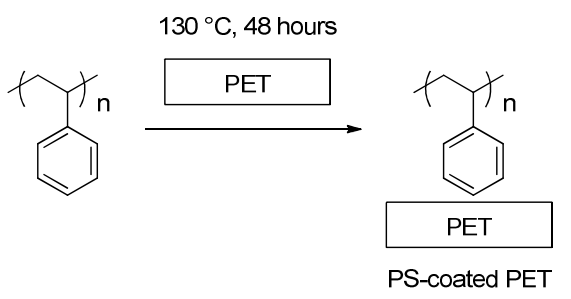

Fig. 3 Preparation of (a) P(S-co-HEMA)-grafted PET, (b) P(S-co-HEMA)-coated PET, and (c) PS-coated PET.

prepare $\mathrm{P}(\mathrm{S}$-co-HEMA)-coated PET, the cleaned PET samples were dipped in an ethyl acetate solution of P(S-co-HEMA) $(0.2 \mathrm{mg} / \mathrm{ml})$ and air-dried (Fig. $3 b)$. To prepare PS-coated PET, the cleaned PET samples were dipped in an ethyl acetate solution of polystyrene $(0.2 \mathrm{mg} / \mathrm{ml})$ and air-dried. They were then thermally annealed at $130^{\circ} \mathrm{C}$ for $48 \mathrm{~h}$ (Fig. 3c).

\section{Instruments and measurements}

${ }^{1} \mathrm{H}$ (500 MHz) NMR spectra were recorded on an AVANCEIII Digital NMR Spectrometer (Bruker Biospin; AV-500) using chloroform-d as solvent. Polymer molecular weights and polydispersities were obtained using a Waters e2695 gel permeation chromatograph equipped with a Viscotek 3580 refractive index detector. Tetrahydrofuran was used as eluent with a flow rate of $1.0 \mathrm{ml} / \mathrm{min}$. The polymers were dissolved in tetrahydrofuran $(2 \mathrm{mg} / \mathrm{ml})$ and the solutions were filtered through $0.45 \mu \mathrm{m}$ membrane filters. The relative molecular weights and polydispersities of the polymers were calculated using polystyrene standards. The FTIR spectra were obtained using a Perkin Elmer FTIR (Spectrum 2000 model) and $\mathrm{NaCl}$ salt windows. Atomic force microscopy (AFM) images were recorded using a Seiko atomic force microscope (Model SPI 4000). The scan speed was $1 \mathrm{~Hz}$. Silicon tips with resonance frequency of $140 \mathrm{kHz}$ and force 
constant of $3.5 \mathrm{~N} / \mathrm{m}$ were used. The chemical composition of the sample surface was investigated using an X-ray photoelectron spectrometer (AXIS ULTRA ${ }^{\text {DLD }}$, Kratos analytical, Manchester UK). The base pressure in the XPS analysis chamber was about $5 \times 10^{-9}$ torr. The samples were excited with $\mathrm{X}$-ray hybrid mode at $700 \times 300 \mu \mathrm{m}$ spot area with a monochromatic $\mathrm{Al}$ $\mathrm{K}_{\alpha 1,2}$ radiation at $1.4 \mathrm{keV}$. The $\mathrm{X}$-ray anode was run at $15 \mathrm{kV}, 10 \mathrm{~mA}$, and $150 \mathrm{~W}$. The photoelectrons were detected with a hemispherical analyser positioned at an angle of $45^{\circ}$ with respect to the sample surface.

\section{RESULTS AND DISCUSSION}

\section{Synthesis of P(S-co-HEMA)}

First, we demonstrated the surface grafting of styrenebased polymers on PET. Styrene was chosen as it is a common monomer for a variety of polymer-related applications. To graft styrene-based polymers onto PET surfaces through the hydroxyl groups, 2-hydroxyethyl methacrylate (HEMA) was used as hydroxylcontaining monomer to provide the linker group in the polymer chain. The copolymer, P(S-co-HEMA), was synthesized by free radical polymerization using benzoyl peroxide as initiator (Fig. 1). To study the effect of copolymer composition on grafting percentage, two copolymers were synthesized. From the integration of peak areas in the NMR spectra, it was found that the two copolymers contained $11 \mathrm{~mol} \%$ and $17 \mathrm{~mol} \%$ of HEMA with respect to styrene contents. By comparing with the FTIR spectra of polystyrene and poly(2-hydroxyethyl methacrylate), the FTIR spectrum of $\mathrm{P}(\mathrm{S}-\mathrm{co}$-HEMA) showed characteristic peaks that belong to a combination of two homopolymers, for example peaks at 3480,1729 , and $1455 \mathrm{~cm}^{-1}$ corresponding to the $\mathrm{O}-\mathrm{H}, \mathrm{C}=\mathrm{O}, \mathrm{C}=\mathrm{C}$ stretches. Hence the FTIR results confirm the structure of the copolymer. From GPC characterization, the numberaverage molecular weights of $11 \mathrm{~mol} \%$ and $17 \mathrm{~mol} \%$ of HEMA-containing copolymers are $15994 \mathrm{~g} / \mathrm{mol}$ and $41492 \mathrm{~g} / \mathrm{mol}$, respectively. The molecular weight distributions of the copolymers are 1.86 and 2.04, respectively. Although these two copolymers possess different molecular weights and polydispersities, previous studies showed that the grafting efficiency does not depend on the polymer's molecular weight or polydispersity ${ }^{17}$. Hence these two copolymers were used for grafting studies as follows.

\section{Effect of surface treatment and copolymer composition}

The PET samples were dipped in an ethyl acetate solution of copolymers and then air-dried for a few min-

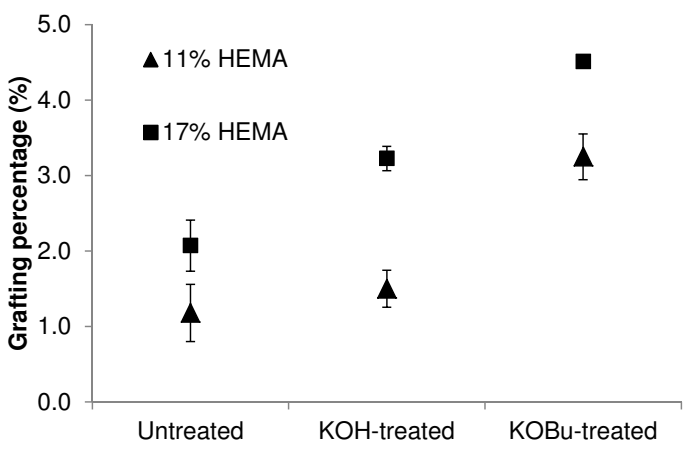

Fig. 4 Grafting percentages of $11 \%$ and 17\% HEMAcontaining copolymers grafted on untreated, $\mathrm{KOH}$-treated, and KOBu-treated PET surfaces.

utes. The copolymer-coated PET samples were then annealed at $130{ }^{\circ} \mathrm{C}$ for $48 \mathrm{~h}$. This annealing condition was first selected because it was similar to that of previous work where HEMA-containing copolymers were grafted on oxide substrates ${ }^{17}$. According to the literature, the grafting process occurs through the condensation reaction between surface hydroxyl groups and hydroxyl groups of the copolymer. The grafting efficiency depends on the hydroxyl contents ${ }^{18}$. Hence the effects of surface treatments and HEMA contents in the copolymer on grafting efficiency were simultaneously investigated. Two copolymers were synthesized as discussed above and three different PET surfaces were prepared: untreated, $\mathrm{KOH}$-treated, and $\mathrm{KOBu}$-treated. The $\mathrm{KOH}$ solution will hydrolyse the ester bonds in the PET chain to create carboxyl and hydroxyl groups whereas the KOBu/ethylene glycol solution generates only the hydroxyl groups on the surface (Fig. 2) ${ }^{22}$. The untreated PET samples theoretically contain the carboxyl and hydroxyl groups only at polymer chain ends. The amount of hydroxyl and carboxyl groups on these surfaces cannot be accurately quantified using routine surface characterization techniques, such as X-ray photoelectron spectroscopy, because the oxygen atoms are also present in the PET polymer chains. The PET surfaces can be, however, qualitatively analysed by contact angle measurement. The water contact angles of untreated, $\mathrm{KOH}$-treated, and KOBu-treated PET samples are $87^{\circ}, 74^{\circ}$, and $75^{\circ}$, respectively. An approximately $12^{\circ}-13^{\circ}$ decrease in water contact angle upon $\mathrm{KOH}$ and $\mathrm{KOBu}$ treatments is consistent with literature results ${ }^{22}$.

The grafting percentage of copolymers on PET was measured (Fig. 4). The results show that both HEMA percentages in the copolymer and the surface treatment used affect the grafting percentage. For both 
copolymers, the KOBu-treated PET samples gave the highest grafting percentage followed by the $\mathrm{KOH}-$ treated samples and untreated samples. These results are expected because the KOBu/ethylene glycol cleaves the ester bonds in PET and converts them into hydroxyl groups whereas the $\mathrm{KOH}$ cleaves the ester bonds into hydroxyl and carboxyl groups. In contrast, the untreated samples have the hydroxyl groups only at polymer chain ends. As a result, the surface hydroxyl contents can be ranked as follows: $\mathrm{KOBu}$-treated $>$ KOH-treated $>$ untreated, which agrees with the grafting percentages. The greater the density of surface hydroxyl groups, the higher the grafting percentage of the copolymers that are grafted on the PET surfaces. The grafting percentage also depends on the HEMA contents in the copolymers. The $17 \%$ HEMA-containing copolymer gave higher grafting percentages than the 11\% HEMA-containing copolymer for all types of PET samples. The higher the number of polymer hydroxyl groups, the higher the grafting percentage of the polymers that are grafted on the surface. These results confirm that the grafting occurs through the hydroxyl groups.

A control experiment was carried out to confirm that the grafting of the copolymers occur through the hydroxyl groups. In this study, polystyrene homopolymer (without HEMA units) was coated and annealed on $\mathrm{KOBu}$-treated PET substrates at $130^{\circ} \mathrm{C}$ for $48 \mathrm{~h}$. The samples were then cleaned using the same procedure as for the copolymers. Since polystyrene does not contain the hydroxyl groups as surface linker, it should be completely removed upon ultrasonication as it is only physically absorbed on the surface. Indeed, after ultrasonication in organic solvent, the grafting percentage was zero, confirming that no polystyrene layer was formed on the PET surface. This result indicates that the hydroxyl groups in the copolymer are the surface linkers necessary for forming chemical bonds with the PET surface.

\section{Effect of annealing temperature}

The effect of annealing temperature on grafting percentage of the copolymer on KOBu-treated PET was investigated. In this study, 17\% HEMA-containing copolymer and KOBu-treated PET substrates were used. The samples were annealed in a hot air oven at temperatures ranging from $100^{\circ} \mathrm{C}$ to $160^{\circ} \mathrm{C}$ for $48 \mathrm{~h}$. As the annealing temperature increased from $100^{\circ} \mathrm{C}$ to $130^{\circ} \mathrm{C}$, the average grafting percentages increased from $1 \%$ to $4 \%$ and then dropped to $2 \%$ when the temperature increased further to $160^{\circ} \mathrm{C}$ (Fig. 5). The thickness of the grafted layer on top and bottom surfaces of the sample was obtained by subtracting

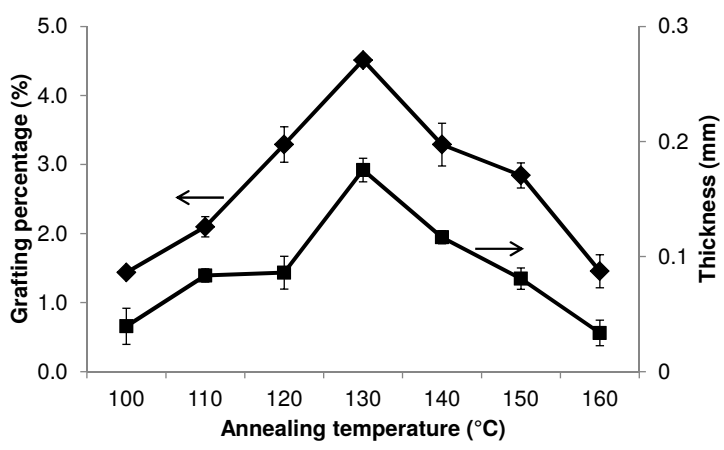

Fig. 5 Grafting percentages (diamonds) and thicknesses (squares) of P(S-co-HEMA) (17 mol\% HEMA content) grafted on KOBu-treated PET samples as a function of annealing temperature.

the thickness of the bare PET sample from the sample thickness after grafting. The thicknesses of the grafted layers correlate well with grafting percentages and reach $0.175 \mathrm{~mm}$ at $130^{\circ} \mathrm{C}$ grafting. High temperatures may decompose the polymer samples resulting in lower grafting percentages. These results suggested that an annealing temperature of $130^{\circ} \mathrm{C}$ is optimal to achieve the highest grafting percentage. A vacuum oven or thermal annealing under inert atmosphere will reduce polymer degradation. However, the aim of this report is to demonstrate the simplicity of this grafting method in which even a home baking oven can be used.

\section{Effect of annealing time}

The effect of annealing time on grafting of the copolymer on PET was studied. Again, 17\% HEMAcontaining copolymer and KOBu-treated PET substrates were used. The samples were annealed at $130^{\circ} \mathrm{C}$, the optimum annealing temperature derived from the previous experiment. The annealing time was varied from $6 \mathrm{~h}$ to $96 \mathrm{~h}$. It was found that an annealing time of $48 \mathrm{~h}$ gave the highest grafting percentage (Fig. 6). The thicknesses of the grafted layers also correlate well with grafting percentages. The shorter annealing times may not be enough to complete the grafting process whereas the longer annealing times may start to decompose the polymer samples.

\section{Surface topography}

The surface topography of KOBu-treated PET and P(S-co-HEMA)-grafted PET samples was measured using atomic force microscopy (AFM). From the AFM images (Fig. 7), it can be observed that the KOBu-treated PET sample was smoother than the $\mathrm{P}(\mathrm{S}$-co-HEMA)-grafted PET sample. The root mean- 


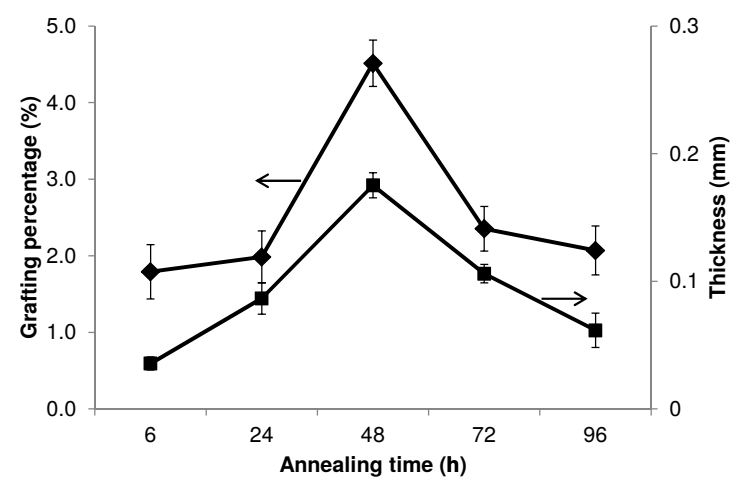

Fig. 6 Grafting percentages (diamonds) and thicknesses (squares) of P(S-co-HEMA) (17 mol\% HEMA content) grafted on $\mathrm{KOBu}$-treated PET samples as a function of annealing time.

(a)

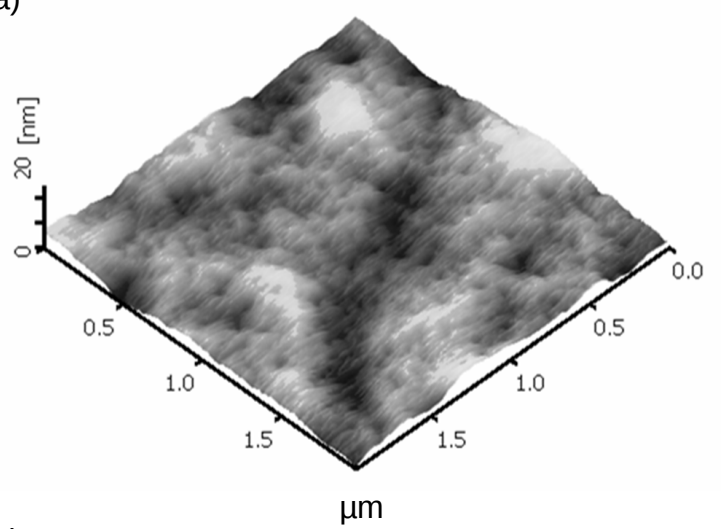

(b)

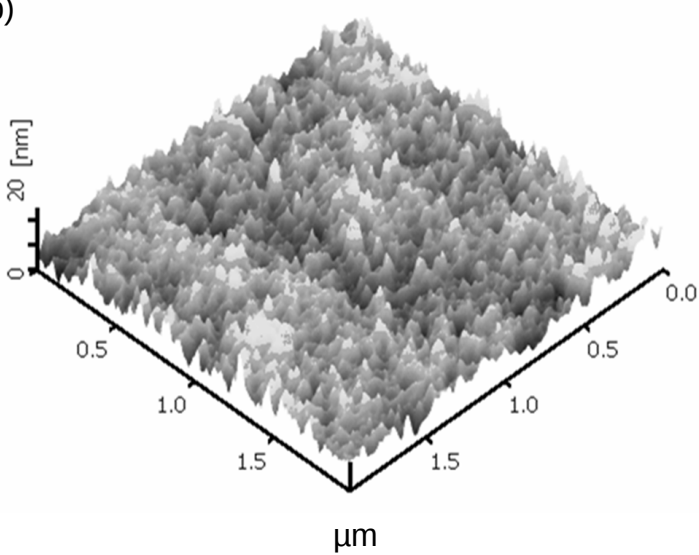

Fig. 7 AFM images of (a) KOBu-treated PET and (b) P(S-co-HEMA)-grafted PET.

square roughness of KOBu-treated PET sample was $1.43 \mathrm{~nm}$ whereas that of the P(S-co-HEMA)-grafted PET sample was $2.98 \mathrm{~nm}$, or about twice as much. The increase in surface roughness of the grafted sample was due to the presence of grafted layer indicating

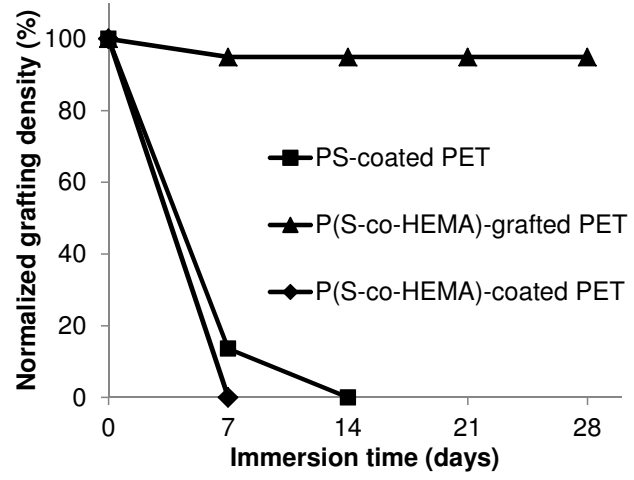

Fig. 8 Plot of normalized grafting percentages of P(S-coHEMA)-grafted PET, P(S-co-HEMA)-coated PET, and PS-coated PET samples in toluene as a function of immersion time.

that the $\mathrm{P}(\mathrm{S}-$-co-HEMA) chains were anchored on the PET surface.

\section{Stability of the polymer layers on PET surface}

The stability of the polymer layers on PET substrates in organic solvent was investigated. In this study, the $\mathrm{P}(\mathrm{S}$-co-HEMA)-grafted PET was prepared by annealing 17\% HEMA-containing copolymer on KOButreated PET at $130^{\circ} \mathrm{C}$ for $48 \mathrm{~h}$. Then the samples were immersed in toluene and compared with the PS-coated PET and P(S-co-HEMA)-coated PET. Toluene was chosen because it is a good solvent for these polymers. The PS-coated PET was prepared by coating polystyrene on KOBu-treated PET samples and then annealed at $130^{\circ} \mathrm{C}$ for $48 \mathrm{~h}$. The samples were then directly immersed in toluene without cleaning. The $\mathrm{P}(\mathrm{S}$-co-HEMA)-coated PET samples were prepared by coating 17\% HEMA-containing copolymer on $\mathrm{KOBu}$-treated PET, but they were not annealed prior to immersion in toluene. The grafting percentages of all samples were monitored overtime and normalized (Fig. 8). It was found that the P(S-co-HEMA)-coated PET and PS-coated PET samples were completely desorbed within 7 and 14 days, respectively, whereas the $\mathrm{P}(\mathrm{S}$-co-HEMA) grafted layer was stable against desorption over 28 days. These results confirm that the presence of HEMA monomer and thermal annealing are necessary for the formation of chemical linkage between the hydroxyl groups which improves the stability of the polymeric layer against desorption in organic solvent.

\section{Synthesis and grafting of P(4VP-co-HEMA)}

One more experiment to demonstrate the scope of this grafting approach was carried out. In this study, 




Fig. 9 XPS spectra of N1s peak of P(4VP-co-HEMA)grafted PET (solid) and KOBu-treated PET (dotted).

$\mathrm{P}(4 \mathrm{VP}-$ co-HEMA) was synthesized using benzoyl peroxide initiator. The copolymer was confirmed using FTIR and NMR characterizations (not shown). Compared with the FTIR spectra of poly(2-hydroxyethyl methacrylate) and poly(4-vinylpyridine), it was found that the copolymer spectrum had characteristic peaks from both homopolymers, for instance, $\mathrm{C}=\mathrm{O}$ at $1725 \mathrm{~cm}^{-1}$ and $\mathrm{C}=\mathrm{C}$ at $1600 \mathrm{~cm}^{-1}$, confirming the formation of the copolymer and consistent with the NMR result.

The KOBu-treated PET samples were dipped in $\mathrm{N}, \mathrm{N}$-dimethylformamide solution of $\mathrm{P}(4 \mathrm{VP}-\mathrm{co}-$ HEMA) and then annealed at $130^{\circ} \mathrm{C}$ for $48 \mathrm{~h}$. After annealing, the samples were ultrasonicated in chloroform. It was found that the grafting percentage of $\mathrm{P}(4 \mathrm{VP}-c o$-HEMA) was $3 \%$, which is comparable to $\mathrm{P}(\mathrm{S}$ - $c$-HEMA).

The XPS spectra of P(4VP-co-HEMA)-grafted PET and KOBu-treated PET were taken (Fig. 9). The N1s peak at $399 \mathrm{eV}$ binding energy was observed whereas the KOBu-treated PET sample did not contain nitrogen. The XPS results confirm the presence of the copolymer layer on the PET surface after thermal annealing.

\section{CONCLUSIONS}

In this work, a simple grafting-to method to prepare thin polymeric layer on PET substrate using thermal annealing was demonstrated. It is a novel result demonstrating that thermal annealing can be used to graft polymers onto polymeric surfaces. Two copolymers, $\mathrm{P}(\mathrm{S}-$-co-HEMA) and $\mathrm{P}(4 \mathrm{VP}-$-co-HEMA) were synthesized and grafted onto PET substrates. The grafting percentage of $\mathrm{P}(\mathrm{S}$-co-HEMA) on PET was affected by surface treatment methods, copolymer composition, annealing temperature, and annealing time. The method offers a number of advantages such as (i) it can be applied to a variety of polymers as long as they contain the hydroxyl linkers and (ii) the grafted polymeric layer shows much improved stability against desorption in organic solvent compared to the physisorbed ones. Based on these results, the grafting method, which can be achieved simply by heating, provides another means for grafting stable polymeric layers onto polymeric substrates. Applications for this grafting strategy include the preparation of functional surfaces, such as superhydrophobic and responsive surfaces. This grafting method is not limited to PET, but is envisioned to be applicable to other polymeric substrates as long as they contain or are prepared to have surface hydroxyl groups.

Acknowledgements: This work was financially supported by the Thailand Research Fund, the Commission on Higher Education (CHE) Grant (MRG5580197), and the Faculty of Science and Technology, Thammasat University. The authors also acknowledge (1) Department of Chemistry and the Central Scientific Instrument Centre, the Faculty of Science and Technology, Thammasat University, for research facilities, (2) the National Metal and Materials Technology Centre for assistance in GPC characterization, and (3) the Department of Physics, Faculty of Science, Chiang Mai University for XPS characterization.

\section{REFERENCES}

1. Solouk A, Cousins BG, Mirzadeh H, Seifalian AM (2011) Application of plasma surface modification techniques to improve hemocompatibility of vascular grafts: A review. Biotechnol Appl Biochem 58, 311-27.

2. Gustafsson Y, Haag J, Jungebluth P, Lundin V, Lim ML, Baiguera S, Ajalloueian F, Del Gaudio C, et al (2012) Viability and proliferation of rat MSCs on adhesion protein-modified PET and PU scaffolds. Biomaterials 33, 8094-103.

3. Mizote N, Katakai A, Tamada M, Matsuoka H (2010) Surface modification of vulcanized rubber by radiation grafting, part 1: Improvement in friction behavior. J Appl Polymer Sci 117, 2825-30.

4. Michielsen S (1999) The effect of grafted polymeric lubricant molecular weight on the frictional characteristics of nylon 6,6 fibers. J Appl Polymer Sci 73, 129-36.

5. Nyström D, Lindqvist J, Östmark E, Hult A, Malmström E (2006) Superhydrophobic bio-fibre surfaces via tailored grafting architecture. Chem Comm 34, 3594-6.

6. Wang Y, Kim JH, Choo KH, Lee YS, Lee CH (2000) Hydrophilic modification of polypropylene microfiltration membranes by ozone-induced graft polymerization. J Membr Sci 169, 269-76.

7. Zdyrko B, Luzinov I (2011) Polymer brushes by the "grafting to" method. Macromol Rapid Comm 32, 859-69.

8. Bao Z, Zheng Y, Baker GL, Bruening ML (2011) 
Preparation of Polymer Brushes Using "Graftingfrom" Techniques. Wiley-VCH, Weinheim, Germany.

9. Onclin S, Ravoo BJ, Reinhoudt DN (2005) Engineering silicon oxide surfaces using self-assembled monolayers. Angew Chem Int Ed 44, 6282-304.

10. Chiba Y, Islam A, Watanabe Y, Komiya R, Koide N, Han LY (2006) Dye-sensitized solar cells with conversion efficiency of 11.1\%. Jpn J Appl Phys 45, L638.

11. Hofer R, Textor M, Spencer ND (2001) Alkyl phosphate monolayers, self-assembled from aqueous solution onto metal oxide surfaces. Langmuir 17, 4014-20.

12. Marczak R, Werner F, Gnichwitz JF, Hirsch A, Guldi DM, Peukert W (2009) Communication via electron and energy transfer between zinc oxide nanoparticles and organic adsorbates. $J$ Phys Chem C 113, 4669-78.

13. Vericat C, Vela ME, Benitez G, Carro P, Salvarezza RC (2010) Self-assembled monolayers of thiols and dithiols on gold: new challenges for a well-known system. Chem Soc Rev 39, 1805-34.

14. Chen Y, Kim H (2009) Preparation of superhydrophobic membranes by electrospinning of fluorinated silane functionalized poly(vinylidene fluoride). Appl Surf Sci 255, 7073-7.

15. Cordeiro AL, Zschoche S, Janke A, Nitschke M, Werner C (2009) Functionalization of poly(dimethylsiloxane) surfaces with maleic anhydride copolymer films. Langmuir 25, 1509-17.

16. You I, Kang SM, Byun Y, Lee H (2011) Enhancement of blood compatibility of poly(urethane) substrates by mussel-inspired adhesive heparin coating. Bioconjugate Chem 22, 1264-9.

17. In I, La YH, Park SM, Nealey PF, Gopalan P (2006) Side-chain grafted random copolymer brushes as neutral surfaces for controlling the orientation of block copolymer microdomains in thin films. Langmuir 22, 7855-60.

18. Paoprasert P, Kandala S, Sweat DP, Ruther R, Gopalan $P$ (2012) Versatile grafting chemistry for creation of stable molecular layers on oxides. J Mater Chem $\mathbf{2 2}$, 1046-53.

19. Paszun D, Spychaj T (1997) Chemical recycling of poly(ethylene terephthalate). Ind Eng Chem Res 36, 1373-83.

20. Hota K, Hota MK, Bera MK, Maiti CK (2012) Flexible metal-insulator-metal capacitors on polyethylene terephthalate plastic substrates. Semicond Sci Tech 27, 105001.

21. Dhahri M, Abed A, Lajimi RH, Mansour MB, Gueguen V, Abdesselem SB, Chaubet F, Letourneur D, et al (2011) Grafting of dermatan sulfate on polyethylene terephtalate to enhance biointegration. J Biomed Mater Res 98A, 114-21.

22. Chen W, McCarthy TJ (1998) Chemical surface modification of poly(ethylene terephthalate). Macromolecules 31, 3648-55. 